\title{
Cell Fate Clusters in ICM Organoids Arise from Cell Fate Heredity \& Division - a Modelling Approach
}

\author{
Tim Liebisch ${ }^{1,}{ }^{*}$, Armin Drusko ${ }^{1}$, Biena Mathew ${ }^{2}$, Ernst H. K. Stelzer ${ }^{2}$, Sabine C. Fischer ${ }^{3}$, and Franziska Matthäus ${ }^{1}$ \\ ${ }^{1}$ Faculty of Biological Sciences \& Frankfurt Institute for Advanced Studies (FIAS), Goethe Universität Frankfurt am Main, \\ Ruth-Moufang-Straße 1, Frankfurt 60438, Germany \\ ${ }^{2}$ Buchmann Institute for Molecular Life Sciences (BMLS) \& Faculty of Biological Sciences, Goethe Universität Frankfurt am Main, \\ Max-von-Laue Str. 15, Frankfurt 60438, Germany \\ ${ }^{3}$ Center for Computational and Theoretical Biology (CCTB), Julius-Maximilians-Universität Würzburg, Campus Hubland Nord 32, \\ 97074 Würzburg, Germany \\ *Correspondence: liebisch@fias.uni-frankfurt.de
}

\section{ABSTRACT}

During the mammalian preimplantation phase, cells undergo two subsequent cell fate decisions. During the first cell fate decision, cells become either part of an outer trophectoderm or part of the inner cell mass. Subsequently, the inner cell mass (ICM) segregates into an embryonic and an extraembryonic lineage, giving rise to the epiblast and the primitive endoderm, respectively. Inner cell mass organoids represent an experimental model system for preimplantation development, mimicking the second cell fate decision taking place in in vivo mouse embryos. In a previous study, the spatial pattern of the different cell lineage types was investigated. The study revealed that cells of the same fate tend to cluster stronger than expected for purely random cell fate decisions. Three major processes are hypothesised to contribute to the final cell fate arrangements at the mid and late blastocysts or $24 \mathrm{~h}$ old and $48 \mathrm{~h}$ old ICM organoids, respectively: 1) intra- and intercellular chemical signalling; 2 ) a cell sorting process; 3) cell proliferation. In order to quantify the influence of cell proliferation on the emergence of the observed cell lineage type clustering behaviour, we developed an agent-based model. Hereby, cells are mechanically interacting with direct neighbours, and exert adhesion and repulsion forces. The model was applied to compare several current assumptions of how inner cell mass neighbourhood structures are generated. We tested how different assumptions regarding cell fate switches affect the observed neighbourhood relationships. The model supports the hypothesis that initial cell fate acquisition is a stochastically driven process, taking place in the early development of inner cell mass organoids. The model further shows that the observed neighbourhood structures can emerge due to cell fate heredity during cell division and allows the inference of a time point for the cell fate decision.

\section{STATEMENT OF SIGNIFICANCE}

Cell fate decisions in early embryogenesis have been considered random events, causing a random cell fate distribution. Using an agent-based mathematical model, fitted to ICM organoid data, we show that the assumed random distribution of cell fates occurs only for a short time interval, as cell fate heredity and cell division quickly lead to spatial cell fate clustering. Our results show that neighbourhood clustering can emerge without specific neighbourhood interactions affecting the cell fate decision. The approach indicates four consecutive phases of early development: 1) co-expression of cell fate markers, 2) cell fate decision, 3 ) division and local cell fate clustering, and 4) phase separation, whereby only the phases 1-3 occur in ICM organoids during the first $24 \mathrm{~h}$ of growth.

\section{INTRODUCTION}

The first steps during mammalian embryo development are ovulation and fertilisation, followed by the preimplantation phase. At this point, the blastocyst is formed, which later implants into the uterus (1). Postimplantation development rapidly proceeds and involves multiple cell differentiation and morphological changes $(1,2)$. The first steps within the complex development processes in mammalian systems involve the cell fate decisions during the preimplantation phase. During development, the preimplantation phase is key to the success of pregnancy in mammals (3). Despite the importance of preimplantation, processes taking place during this phase are not fully understood. 
The mouse is a common model organism to study the preimplantation phase. The 8 to 16 cells morula is formed until embryonic day 2.5 (E2.5) after fertilisation. At this stage, the first cell fate decision is taking place. Cells on the surface of the morula become trophectoderm (TE), while cells inside the morula are forming the inner cell mass (ICM) $(4,5)$. During E3.0 to E4.5, the second cell fate decision process takes place: ICM segregates into epiblast (Epi) and primitive endoderm (PrE) (6-8). NANOG and GATA6 are described as the first markers for Epi and PrE segregation, respectively. Expression levels of NANOG and GATA6 undergo progressive changes during the morula stage and the early blastocyst $(9,10)$. In early blastocysts (E3.0), all ICM cells seem to co-express NANOG and GATA6 $(7,11,12)$. Subsequently, NANOG and GATA6 are gradually up- or down-regulated during the 32-cell stage. Thereby, both transcription factors repress each other locally $(10,13-17)$, leading to a mutually exclusive transcription factor expression in late blastocysts (64 cells) (18). Once a cell-fate is determined it is only possible to switch the fate by an external modulation of the included signalling pathways $(16,17,19)$. While at early phases of preimplantation, the spatial distribution of NANOG and GATA6 positive cells is commonly described as a random salt-and-pepper pattern, GATA6 positive cells are sorted to the rim of the ICM in the late blastocyst and NANOG positive cells are forming an inner core $(1,7,10,11,14)$.

Three major processes contribute to the final cell fate arrangements in mid and late blastocysts (reviewed in (20)). (1) Inter- and intracellular chemical signalling: the influence of NANOG, GATA6, and the FGF/ERK pathway onto the cell fate acquisition of single cells have been heavily studied over the past years $(10,13-15,17,19,21-27)$. (2) Cell sorting processes: since cell lineage tracking revealed that ICM cells do not change their cell fate at E3.5, a cell sorting process is leading to the observed cell fate arrangements in late blastocyst (7, 10, 28-31). (3) Cell proliferation: from early blastocyst (E3.0) to late blastocyst (E4.5) it takes two to three rounds of cell divisions. Cell proliferation is hypothesised to contribute to the segregated state at E4.5 (32-34).

A recent study introduced a novel 3D stem cell system named ICM organoids (in the following the term organoid refers to the biological system, while the term spheroid is used in context of modelled data), which is based on inducible mouse embryonic stem cells (35). ICM organoids mimic the segregation into Epi and PrE without forming a TE and reproduce key events and timing of cell fate specification and cell-cycle progression in the ICM of mouse blastocysts. Thus, ICM organoids provide a powerful tool to develop and test biological preimplantation hypotheses in vitro. The cell fate of the cells within the ICM organoids are determined by their expression level of the transcription factors NANOG and GATA6. In total, four different cell types are described: mostly NANOG expressing cells which express a small amount of GATA6 $\left(\mathrm{N}_{+} \mathrm{G}_{-}\right)$, mostly GATA6 expressing cells which express a small amount of NANOG $\left(\mathrm{N}_{-} \mathrm{G}_{+}\right)$, cells that express NANOG and GATA6 on a high level $\left(\mathrm{N}_{+} \mathrm{G}_{+}\right)$and cells that express both transcription factors at a low level (N-G_. $)$. After $24 \mathrm{~h}$ of growth, most ICM organoid cells are either $\mathrm{N}_{+} \mathrm{G}_{-}$or $\mathrm{N}_{-} \mathrm{G}_{+}$, meaning most ICM organoid cells are expressing one of both transcription factors at a high level and the other one at a low level, respectively. The spatial segregation into an inner core of $\mathrm{N}_{+} \mathrm{G}_{-}$cells and an outer layer of $\mathrm{N}_{-} \mathrm{G}_{+}$cells is visible after $48 \mathrm{~h}$ of growth. However, in contrast to mid mouse blastocysts, which consist of approximately 64 cells, ICM organoids comprise over 400 cells after $24 \mathrm{~h}$ of growth and more than 1000 cells after $48 \mathrm{~h}$ of growth. In order to quantify the patterns of neighbourhood distributions, a neighbourhood analysis of $\mathrm{N}_{+} \mathrm{G}_{-}$cells, $\mathrm{N}_{-} \mathrm{G}_{+}$cells, $\mathrm{N}_{+} \mathrm{G}_{+}$cells and $\mathrm{N}_{-} \mathrm{G}_{-}$cells was conducted in ICM organoids (35). Interestingly, the study revealed a local clustering of cells sharing the same expression type, inconsistent with the assumed salt-and-pepper pattern of cell fates.

The work of Mathew et al. (2019) (35) indicates that between the two stages of an initial salt-and-pepper distributed cell fate acquisition (E3.0-E3.5 early-blastocyst or $0 \mathrm{~h}$ old ICM organoids) and the final segregation of different cell fates (E4.5 late blastocyst or $48 \mathrm{~h}$ old ICM organoids), a local clustering of cell fates arises (E3.75 mid blastocyst or $24 \mathrm{~h}$ old ICM organoids). In order to test whether this pattern can be achieved through simple rules, static models were used. In these models, the cells are assigned to a cell fate but do not move. Different simple rules for these cell fate assignments were tested. However, none of the tested rules could reproduce the observed neighbourhood pattern of local clustering in $24 \mathrm{~h}$ old ICM organoids (35).

The purpose of this study is to investigate to which amount the observed neighbourhood structure in ICM organoids can be explained just considering cell divisions. To this end, a mathematical model is implemented. The implemented model is a 3D agent-based model. Agent-based models provide a technique to represent a wet-lab experiment under idealised conditions (36). Comparable agent-based models are commonly used to study cancer growth, cell proliferation or the contribution of single cells towards collective cell migrations (37-42). The model is given as a set of differential equations, describing mechanical cell-cell interactions, such as adhesion and repulsion forces, cell growth and cell division involving cell fate heredity. It is assumed that the initial cell fate acquisition results in a salt-and-pepper distribution at E3.5, which eventually leads to two segregated populations at E4.5 $(10,11,29,43)$. Hence, the initial cell fate decision is modelled as a stochastic process (omitting a detailed description of the signalling pathway dynamics) (44-46).

We use the model to investigate the hypothesis that the observed local cell fate clustering in $24 \mathrm{~h}$ old ICM organoids can arise from cell divisions alone, whereby cell fates are (partially) passed on to both daughter cells. Simulations were conducted under four hypotheses, each considering different cell fate switch rates during cell division. Our results indicate that the observed cell fate clustering can indeed be explained as a randomly distributed cell fate decision with subsequent divisions and cell fate 
heredity. Furthermore, based on the neighbourhood statistics, a time point for the cell fate decision (prior to the $24 \mathrm{~h}$ growth stage) can be inferred.

\section{MATERIALS AND METHODS Experimental data}

Our study is based on experimental results from Mathew et al. (2019) (35). They used a system based on mouse embryonic stem cells which allows differentiation into primitive endoderm like cells (26). After induction of differentiation, 200 of these cells were placed in a nonadhesive well and centrifuged. Subsequently, the cells formed the 3D ICM organoids. This experimental approach implies that the cell fates in the initial organoid, composed of about 200 cells, are randomly distributed.

In $24 \mathrm{~h}$ and $48 \mathrm{~h}$ old ICM organoids, expression levels for NANOG and GATA6, as well as the nuclei positions for all cells were determined by fluorescence microscopy and subsequent image analysis. In total, four different expression types were established: NANOG and GATA6 expressing cells (double positive $\mid \mathrm{N}_{+} \mathrm{G}_{+}$), cells that express both transcription factors at a low level (double negative | N_G - $_{-}$, cells that express NANOG at a high cells and GATA6 at a low level $\left(\mathrm{N}_{+} \mathrm{G}_{-}\right)$and cells that express GATA6 at a high level and NANOG at a low level $\left(\mathrm{N}_{-} \mathrm{G}_{+}\right)$.

In the following, the stage of $24 \mathrm{~h}$ old and $48 \mathrm{~h}$ old ICM organoids will be referred to as $t_{1}$ and $t_{2}$, respectively. During the simulations, cell fates and positions were recorded when the cell count in the simulation coincided with the average ICM organoid cell count in the experiment at $t_{1}(441$ cells at $24 \mathrm{~h})$ and $t_{2}(1041$ cells at $48 \mathrm{~h})$.

\section{Model implementation}

An individual cell-based model that defines a small set of cellular features, implemented by Stichel et al. (2017) (42), is extended to explain the rise of local cell fate clustering in ICM organoids. The model describes the displacement of cells in response to external forces exerted by surrounding cells:

$$
\mathbf{F}_{i, k}=F_{0} \cdot F\left(r_{i}, r_{k},\left\|\mathbf{x}_{i}-\mathbf{x}_{k}\right\|\right) \cdot \frac{\mathbf{x}_{i}-\mathbf{x}_{k}}{\left\|\mathbf{x}_{i}-\mathbf{x}_{k}\right\|}
$$

with $F_{0}$ a positive constant, representing the strength of the mechanical interaction, $r_{i}$ the radius of the $i$ th cell, $\mathbf{x}_{i}=\left(x_{i}, y_{i}, z_{i}\right)$ the position of the $i$ th cell and

$$
F\left(r_{i}, r_{k}, d\right)=\left\{\begin{array}{ll}
2 \cdot\left(e^{-2 a\left(d-\left(r_{i}+r_{k}\right)\right)}-e^{-a\left(d-\left(r_{i}+r_{k}\right)\right)}\right) & d<\sigma r_{i} \\
0 & d \geq \sigma r_{i}
\end{array} .\right.
$$

As given by the Morse potential (Eq. 2), the force between two cells is positive (repulsive), if the distance between the cell centres is below the sum of their radii, and negative (attractive) for $\left(r_{i}+r_{k}\right)<d<\sigma r_{i}$. Repulsion accounts for the limited compressibility of cells, while attraction accounts for cell-cell adhesion. The attractive part of the potential is cut off for cells at a distance above $\sigma r_{i}$. If the distance of two cells equals the sum of their radii they are in perfect distance and thus neither exert attraction nor repulsion onto each other. The elasticity of the cells is given by the parameter $a$. The stiffness of cells increases with $a$. Displacement of cells in this model is only determined by forces exerted on them:

$$
\frac{d \mathbf{x}_{i}}{d t}=\sum_{k, k \neq i} \mathbf{F}_{i, k}
$$

Each cell in this model is described by three features. A position $\mathbf{x}$, a radius $r$ and an expression type $\epsilon$. Other model parameters are assumed to be the same for all cells (e.g. elasticity, adhesion strength). The radius (size) of the cells is growing over time with

$$
\frac{d r_{i}}{d t}=k \cdot\left(r^{*}-r_{i}\right)
$$

with $k$ a (positive) growth constant and the maximum cell size $r^{*}$. Cell division is determined by a stochastic process which depends on the cell radius but not on the cell type. During cell division, the cell volume is preserved. The mother cell keeps its position $\left(\mathbf{x}_{m}\right)$ and reduces its radius $\left(r_{m}\right)$ with: 


$$
r_{m, n e w}=r_{m} \cdot \sqrt[3]{\frac{1}{2}}
$$

The daughter cell $\left(\mathbf{x}_{d}\right)$ is generated close to $\mathbf{x}_{m}$, with $\mathbf{x}_{d}=\mathbf{x}_{m}+\xi$ with $\xi$ a random 3D vector $\left(\delta_{x}, \delta_{y}, \delta_{z}\right)$ containing small values $\left(\delta<<r_{m, n e w}\right)$. The daughter cell is assigned the same size as the mother cell $\left(r_{d}=r_{m, n e w}\right)$. The factor conserves the total cell volume during cell division. Directly after cell division, both cells are growing as given by Eq. 4 and change their positions as given by Eq. 3 .

Since it was shown that the initial cell fate decision can be described as a stochastic process (44-46) and because the experimental approach leads to a random assembly of cell types, the initial expression type $\epsilon$ of the simulated cells is assigned randomly from the four expression types

$$
\epsilon \in\left\{\mathrm{N}_{-} \mathrm{G}_{-}, \mathrm{N}_{+} \mathrm{G}_{+}, \mathrm{N}_{+} \mathrm{G}_{-}, \mathrm{N}_{-} \mathrm{G}_{+}\right\} \text {. }
$$

All parameters and the chosen values for the model are shown in Table A1.

\section{Cell fate heredity}

The model can be used to test several assumptions addressing cell fate heredity. In total, four different hypotheses are tested. We consider the cell fate switches shown in Fig. A1 (i.e. N-G remain N-G. or become $\mathrm{N}_{+} \mathrm{G}_{+} ; \mathrm{N}_{+} \mathrm{G}_{+}$remain $\mathrm{N}_{+} \mathrm{G}_{+}$or become $\mathrm{N}_{+} \mathrm{G}_{-}$ or $\mathrm{N}_{-} \mathrm{G}_{+} ; \mathrm{N}_{+} \mathrm{G}_{-}$and $\mathrm{N}_{-} \mathrm{G}_{+}$remain $\mathrm{N}_{+} \mathrm{G}_{-}$and $\mathrm{N}_{-} \mathrm{G}_{+}$or switch to the opposite cell fate).

Hypothesis $1(\mathrm{H} 1)$ is considering no cell fate switches at all. The cell fate of a cell is strictly passed on to its daughter cells during cell division. Hypothesis $2(\mathrm{H} 2)$ assumes that $\mathrm{N}_{+} \mathrm{G}_{+}$produces $\mathrm{N}_{+} \mathrm{G}_{+}$and $\mathrm{N}_{-} \mathrm{G}_{+}$in equal parts during cell divisions, such that the amount of $\mathrm{N}_{+} \mathrm{G}_{+}$remains constant over time, while the relative amount of $\mathrm{N}_{-} \mathrm{G}_{+}$is increasing. We assume a third hypothesis $(\mathrm{H} 3)$, with parameters such that the proportion transitions from $t_{1}$ to $t_{2}$ both are fitted. With a fourth hypothesis (H4) we consider a small cell fate transition rate from $\mathrm{N}_{-} \mathrm{G}_{+}$to $\mathrm{N}_{+} \mathrm{G}_{-}$. The parameters and the chosen values for all hypotheses are shown in Tab. A2.

Please note that the fit involves only cell proportion data, and not the spatial positioning.

\section{Model framework}

The implemented model is illustrated by a flowchart in Fig. A2. For all hypotheses, the initial cell fate proportion has been determined and assigned to occur at an earlier time point $\left(t_{0}\right)$. In particular, we couple $t_{0}$ to the cell count and initiate the cell fate when the simulated ICM spheroid reached a cell count of 200, 300 or 400 cells. We determine the initial proportions of the cell fates by proportion data at $t_{1}$ taken from the experimental data, omitting the spatial component. Using the system of linear differential equations (Eq. A1-A3), the theoretical proportions at $t_{0}$ are determined. After cell fate acquisition, the simulation proceeds until $t_{1}$ and $t_{2}$ are reached. Both time points are coupled to cell counts as well (i.e. 441 cells at $t_{1}$ and 1041 cells at $t_{2}$ ). When $t_{1}$ and $t_{2}$ are reached, the model saves the spatially resolved cell positions and cell fates as output. Each simulation is repeated 100 times.

\section{Neighbourhood analysis and statistics}

Cell neighbours were determined for both, simulation and experimental data, using the Delaunay triangulation. For the neighbourhood statistics, we derived the set of all neighbours of all cells of a given fate $j$, and computed the proportion of cell types based on this neighbourhood set. Neighbourhood proportions were collected for every executed simulation. For statistical comparison between experimental and simulated data according to single neighbourhood structures, we used the Wilcoxon-Mann-Whitney test with Bonferroni correction for multiple testing. In order to compare the overall fit of the simulated pattern to experimental data, we used the effect size as the relative deviation $(\psi)$ as given in (35).

$$
\psi=\frac{|(\bar{s}-\bar{m})|}{\bar{m}},
$$

with $\bar{s}$ representing the mean of the simulated data and $\bar{m}$ the mean of the experimental data. 


\section{Spatial analysis of expression type distribution}

During visual inspection of the biological data, we noticed that double negative cells often clustered at the rim of the ICM organoids. In order to visualise this effect, the following procedure was applied to experimental data for $24 \mathrm{~h}$ old and $48 \mathrm{~h}$ old ICM organoids. We assumed the spatial heterogeneity to be the same in small and in large ICM organoids, and therefore we normalised the size of all ICM organoids to equalise them in space. For normalisation, the median absolute divergence is used instead of the standard deviation because it is more robust with respect to outliers. The centre of mass for double negative cells was determined and the entire ICM organoid was rotated such that the centre of mass was located on the x-axis (i.e. $x>0, y=0, z=0)$. The rotated cell positions were combined in one data set. Each double negative cell was assigned the value 1 and the value 0 was assigned to all other cells. Interpolation on the combined data generated a continuous clustering pattern from experimental data. In the interpolated dataset, the label 1 indicated the presence of a particular expression type, while 0 indicated its absence. The procedure was repeated for double positive, NANOG positive and GATA6 positive cells to show that the visualised heterogeneity is not an artefact resulting of this procedure.

In order to analyse the cell fate proportions in dependence on their relative distance to the ICM organoid centre, we measured the distance of the cells of each ICM organoid to their respective centre of mass. Subsequently, we divided these distances into ten intervals. Eventually the mean proportions and standard deviations for the ten intervals were determined for all $24 \mathrm{~h}$ old ICM organoids and $48 \mathrm{~h}$ old ICM organoids. Unless otherwise stated, the model and data analysis methods were implemented in MATLAB R2019b.

\section{RESULTS}

The purpose of the simulations was to study, if the complex 3D neighbourhood pattern in the ICM organoids can be explained by cell divisions alone. To this end, we combined several simple mechanisms, i.e. cell mechanics, a stochastic expression type assignment and cell fate heredity during cell division, but omitted cell type segregation processes, intra- and extracellular signalling.

We show that the simulations can be used to predict the ICM organoid cell count when the cell fate decision occurs. For this reason, we used a mechanistic mathematical model for 3D spheroid growth in order to investigate the distribution pattern of cell fates in $24 \mathrm{~h}$ old ICM organoids (see Methods). During the simulation, the cells were assigned to the different expression types (i.e. $\mathrm{N}_{-} \mathrm{G}_{-}, \mathrm{N}_{+} \mathrm{G}_{+}, \mathrm{N}_{+} \mathrm{G}_{-}$and $\mathrm{N}_{-} \mathrm{G}_{+}$) randomly at $t_{0}$, whereby the cell type proportions were set to fit the experimental data for $24 \mathrm{~h}$ old ICM organoids. The time point for the initial expression type assignment $\left(t_{0}\right)$ was coupled to cell count of the spheroid (200, 300 or 400 cells). One $24 \mathrm{~h}$ old ICM organoid, one $48 \mathrm{~h}$ old ICM organoid, as well as simulated ICM spheroids for $t_{1}$ and $t_{2}$ are shown in Fig. 1 A.

We tested four hypotheses addressing different cell fate heredity strategies (see Methods). In assumption H1, each cell passes on its cell fate to both daughter cells. For assumption $\mathrm{H} 2, \mathrm{~N}_{+} \mathrm{G}_{+}$are allowed to give rise to $\mathrm{N}_{+} \mathrm{G}_{+}$or $\mathrm{N}_{-} \mathrm{G}_{+}$cells. The production of $\mathrm{N}_{-} \mathrm{G}_{+}$cells from $\mathrm{N}_{+} \mathrm{G}_{+}$cells is sensible, because a) a switch from $\mathrm{N}_{+} \mathrm{G}_{-}$to $\mathrm{N}_{-} \mathrm{G}_{+}$or vice versa is biologically not feasible $(16,17), \mathrm{b})$ the amount of $\mathrm{N}_{+} \mathrm{G}_{+}$cells remains constant between the time points $t_{1}$ and $t_{2}$, and c) the proportion of $\mathrm{N}_{-} \mathrm{G}_{+}$ cells increases strongly from $t_{1}$ to $t_{2}$. Although cell fate switches from $\mathrm{N}_{+} \mathrm{G}_{-}$to $\mathrm{N}_{-} \mathrm{G}_{+}$or vice versa are biologically not feasible we allow them in $\mathrm{H} 3$ and $\mathrm{H} 4$ in order to fit the proportion data measured at $t_{2}$. H3 allows cell fate switches from $\mathrm{N}_{+} \mathrm{G}_{-}$to $\mathrm{N}_{-} \mathrm{G}_{+}$ and $\mathrm{H} 4$ considers a small flux between $\mathrm{N}_{+} \mathrm{G}_{-}$and $\mathrm{N}_{-} \mathrm{G}_{+}$cells (see Fig. A1 and Tab. A2).

To determine the hypotheses with the closest fit to the experimental data, the effect size as the relative deviation of the simulated data is calculated (see Fig. A3). H1 and H2 show the closest fit to the experimental data (see Fig. 1). The results for $\mathrm{H} 3$ and $\mathrm{H} 4$ are shown in the supplement (see. Fig. A4).

Starting from the initial cell fate assignment at $t_{0}$, cell growth, displacement according to adhesion and repulsion forces, and cell divisions were simulated according to Eqs. 1-6. Cell fates and positions were recorded at $t_{1}$ and $t_{2}$.

The expression type compositions of simulated ICM spheroids were determined and statistically compared with experimental data using the Wilcoxon-Mann-Whitney test with Bonferroni correction. For H1 (Fig. 1 B) and also for H2 (Fig. 1 C), the simulated proportions agreed very well with the experimental data at $t_{1}$. At $t_{2}$, the simulated proportion data showed for the assumption $\mathrm{H} 1$ significant differences to experimental data for all expression types at all cell fate initialisation time points $t_{0}(p<0.05)$. For the assumption $\mathrm{H} 2$ (Fig. $1 \mathrm{C}$ ), significant differences between predicted and experimental data at $t_{2}$ were determined for cells with the expression types $N_{-} G_{-}, N_{+} G_{-}$and $N_{-} G_{+}(p<0.05)$. No statistically significant differences were found between simulated and experimental data for cells with the expression type $\mathrm{N}_{+} \mathrm{G}_{+}$at $t_{2}$.

The neighbourhood distribution for H1 (see Fig. 1 D) largely agreed with the experimental data at $t_{1}$, the only exception being the neighbourhood statistics involving N.G. cells. Overall, the neighbourhood distributions fitted the experimental data best, if the cell fate assignment occurred at $t_{0}=200$ (lowest $\psi$-value, see Fig. A5). Concerning the neighbourhood structures measured at $t_{2}$, the prediction power of the model decreased strongly (see Fig. A4 A).

The neighbourhood statistics for $\mathrm{H} 2$ agreed reasonably well with the experimental data (see Fig. 1 E). The neighbourhood 
pattern of cells with the expression types $\mathrm{N}_{-} \mathrm{G}_{-}$and $\mathrm{N}_{+} \mathrm{G}_{-}$at $t_{1}$ were similar to the neighbourhood structures obtained under assumption $\mathrm{H} 1$, including the misfit involving $\mathrm{N}_{-} \mathrm{G}_{-}$cells. In addition, simulated $\mathrm{N}_{+} \mathrm{G}_{+}$cells were significantly less often neighbours of other $\mathrm{N}_{+} \mathrm{G}_{+}$cells than the experimental data suggests. The latter speaks against a cell fate change of $\mathrm{N}_{+} \mathrm{G}_{+}$cells into $\mathrm{N}_{-} \mathrm{G}_{+}$cells.

If performed under the assumption of the $\mathrm{H} 2$ model, the evaluated patterns for $t_{2}$ showed a better agreement with the experimental data. In particular, the predicted neighbourhood statistics of $\mathrm{N}_{+} \mathrm{G}_{+}$cells differ statistically less significantly from the in vitro measured patterns compared to the simulated cell neighbourhood statistics under the assumption H1. The simulated neighbourhood structures are shown in the Supplementary Material (see. Fig A4B).

According to the overall comparison of neighbourhood structures, the best agreement between experimental data and simulation required $t_{0}=200$ cells. This implies that all or most cells acquired a cell fate prior to the composition into the organoid, and that after the assembly into a 3D multicellular system, only cell division and cell sorting took place.

\section{Spatial analysis of expression type distribution}

Simulation results of the model hypotheses $\mathrm{H} 1$ and $\mathrm{H} 2$ fitted well to the experimental data on the spheroid expression type composition, and could also explain the neighbourhood statistics to a large degree. The only disagreement between simulation and experiment concerned the neighbourhood statistics of N-G. cells. In order to investigate the reasons for this disagreement, we conducted an additional spatial analysis of the expression type distribution. Fig. 2 shows 3D views of the average ICM organoid composition for both time points $t_{1}$ and $t_{2}$, marking the spatial density of a given cell fate $j$ (see Methods). Cells with the expression type $\mathrm{N}_{+} \mathrm{G}_{+}$were spread evenly over the whole ICM organoid at $t_{1}$ and $t_{2}$. The same distribution pattern was obtained for $\mathrm{N}_{+} \mathrm{G}_{-}$and $\mathrm{N}_{-} \mathrm{G}_{+}$expression type cells at $t_{1}$. Their spatial distribution pattern changed over time. At $t_{2}, \mathrm{~N}_{+} \mathrm{G}_{-}$cells formed a cluster in the centre of the ICM organoid while $\mathrm{N}_{-} \mathrm{G}_{+}$cells formed an evenly distributed outer layer around the inner core of the ICM organoid. Concerning the N_G. cells, we find that this expression type tended to be positioned in the outer parts of the ICM organoid at both time points, $t_{1}$ and $t_{2}$. In both cases, their distribution was unevenly spread over the outermost layer of cells in the ICM organoid.

\section{DISCUSSION}

In this study, we investigated whether cell division and cell fate heredity alone can account for cell fate clustering and experimentally observed 3D neighbourhood statistics measured in $24 \mathrm{~h}$ and $48 \mathrm{~h}$ old ICM organoids, resembling the mid mouse blastocysts (E3.75 to E4.5). Generally, three major processes are hypothesised to lead from undifferentiated ICM cells at E3.0 to the final cell fate arrangement at E4.5 (i.e. inter- and intracellular chemical signalling, cell sorting processes and cell proliferation, reviewed in (20)). We demonstrated that a simple model, involving mechanical interactions such as adhesion and repulsion, cell division with cell fate heredity, and a stochastically driven cell fate, can explain the complex 3D neighbourhood statistics in $24 \mathrm{~h}$ old ICM organoids. The proposed model is based on a minimal number of assumptions: (1) Up to a certain time point, when the simulated ICM spheroid consists of a predetermined number of cells (i.e. $t_{0}$ ), all cells coexpress the transcription factors NANOG and GATA6, analogous to mouse embryonic development to E3.0 $(11,45)$. (2) At $t_{0}$, a cell fate is introduced to each cell with a given probability (omitting a detailed description of the signalling pathway dynamics) $(45,46)$. Cells either continue to co-express both transcription factors $\left(\mathrm{N}_{+} \mathrm{G}_{+}\right)$, stop the expression of one of them $\left(\mathrm{N}_{+} \mathrm{G}_{-}\right.$or $\left.\mathrm{N}_{-} \mathrm{G}_{+}\right)$, or stop to express both (N-G.). The probabilities for each case are extracted from experimental data (35). (3) Once a certain cell fate has been chosen, cells pass on their cell fate to the daughter cells during cell division according to certain rules (see Fig. A1). Four hypotheses addressing cell fate switches during cell division were tested: The cell fate is passed on to both daughter cells, switches between different fates are not allowed (H1); $N_{+} G_{+}$cells give rise to $N_{+} G_{+}$or $N_{-} G_{+}$cells $(H 2) ; N_{+} G_{+}$cells give rise to $\mathrm{N}_{+} \mathrm{G}_{+}, \mathrm{N}_{+} \mathrm{G}_{-}$or $\mathrm{N}_{-} \mathrm{G}_{+}$cells and $\mathrm{N}_{+} \mathrm{G}_{-}$can switch to $\mathrm{N}_{+} \mathrm{G}_{-}(\mathrm{H} 3)$; H3 is extended, such that $\mathrm{N}_{+} \mathrm{G}_{-}$cells can switch to $\mathrm{N}_{+} \mathrm{G}_{-}$ and vice versa $(\mathrm{H} 4)$.

\section{Proportions}

Since the initial cell fate assignment at different $t_{0}$ was adjusted to fit the proportion data known from $t_{1}$, all models reproduced the proportions for $t_{1}$ very well.

At $t_{2}, \mathrm{~N}_{+} \mathrm{G}_{-}$cells represented the majority in the ICM organoid. The increase of $\mathrm{N}_{-} \mathrm{G}_{+}$cells from $t_{1}$ to $t_{2}$ can be explained by either cell fate switches from $\mathrm{N}_{+} \mathrm{G}_{-}$to $\mathrm{N}_{-} \mathrm{G}_{+}$or by an enhanced cell division rate for $\mathrm{N}_{-} \mathrm{G}_{+}$cells, for instance due to an interaction between the cell growth rate and mechanical forces exerted by surrounding cells. The first can be considered as unlikely, regarding the overall fit of the simulated patterns to experimental data (see Fig. A3). These results indicate that cell fate switches from $N_{+} G_{-}$to $N_{-} G_{+}$and vice versa are unlikely, which is in agreement with the literature $(16,17,19)$.

Cells on the surface of ICM organoids might grow faster than cells in the centre of ICM organoids, where the cell density is 
high $(37,47) . \mathrm{N}_{-} \mathrm{G}_{+}$expressing cells are predominantly found at the rim of ICM organoids where the cell density is lower, which might result in a higher cell division rate $(24,48)$. A feedback of pressure onto cell growth is not included in our model, hence this aspect has not been captured.

\section{Neighbourhood structures}

Complex 3D spatial arrangements of cells with particular cell fates characterise early stages of PrE and Epi segregation. ICM organoids mimic those cell arrangements after $24 \mathrm{~h}$ of growth and after $48 \mathrm{~h}$ of growth for mid and late mouse blastocysts, respectively. Mathew et al. (2019) (35) used a computational rule-based static model in order to reproduce the cell fate pattern measured in $24 \mathrm{~h}$ old ICM organoids. Hereby, four different hypotheses have been tested. The first three simulations were based on hypotheses derived from the salt-and-pepper pattern, while the fourth tested pattern was based on a local density. Patterns from all four simulations were significantly different from experimentally observed patterns (35), in particular because the clustering of the cells sharing the same cell fate could not be reconciled with any of the tested models.

In this study, we showed that an initial salt-and-pepper distributed cell fate decision, in addition to cell division involving cell fate heredity, adhesion and mechanical repulsion, can explain the lineage composition and spatial distribution of $\mathrm{N}_{+} \mathrm{G}_{-}$and $\mathrm{N}_{+} \mathrm{G}_{-}$cells in $24 \mathrm{~h}$ old ICM organoids. The main assumption here was that the $24 \mathrm{~h}$ time point did not represent the time point of the cell fate decision. Instead, we assumed that the cell fate decision occurred previously, and that the local clustering of cell fates arises from cell divisions. Under this hypothesis, we found that the assumption $\mathrm{H} 1$ as well as the assumption $\mathrm{H} 2$ showed good agreement with experimental data for $24 \mathrm{~h}$ old ICM organoids, whereby the assumption $\mathrm{H} 1$ reflected the neighbourhood structure of $\mathrm{N}_{+} \mathrm{G}_{+}$cells better than the assumption $\mathrm{H} 2$. With increasing complexity of the cell fate heredity rules, the overall goodness of the fit of the simulated compared to the experimentally determined neighbourhood statistics decreases. Hence, the experimental data is explained best, if the cell fates are determined at $t_{0}$, and a phase of cell divisions follows.

A comparable model, which is also based on mechanic cell displacement and cell division, but takes also into account an intercellular network for cell fate decisions was used in (41) to predict cell fate decisions in in vivo mouse blastocysts. According to their findings, the spatio-temporal distribution of PrE and Epi cells follows the salt-and-pepper pattern, whereby Epi cells are preferentially surrounded by PrE cells and vice-versa (41).

The distribution of cell fate patterns is questioned by several recent studies that are highlighting the need of neighbourhood interactions for the rise of Epi and PrE lineages $(25,27,41)$. However, we hypothesise that the local spatial clustering of cell fates as obtained in (35) might not be indicative of specific neighbourhood interactions involved in cell fate decision. We further hypothesise that a cell fate clustering is a transient state, characteristic of blastocysts and ICM organoids in the time span between cell fate decision and spatial sorting. In particular, our results indicate that an initial cell fate decision based on a random distribution, in addition to cell divisions and cell fate heredity, can give rise to local cell clusters, exhibiting the same neighbourhood statistics as observed in experiments (35).

With the given restrictions on the cell fate switches, it was not possible to fit the proportion transition from $24 \mathrm{~h}$ to $48 \mathrm{~h}$ data perfectly well. Although cell fate switches are considered unlikely, we investigated if the proportion data and neighbourhood structures could be approximated better if further cell fate switches are allowed. Indeed, this relaxation allows for a better fit of the cell proportion data. However, the neighbourhood statistics are not approximated well under the hypotheses H3 and H4 (see Supplementary Material).

Using the model, we can provide an estimate for the time point when the cell fate decision process in ICM organoids is finalised. Since the simulated data fits best to the experimental data when the cell fate is introduced at a cell count of 200 cells, we assume that in vitro, most cells of the ICM organoids acquired a cell fate after the composition into the organoid and before their first cell division.

This implies that the initial cell fate acquisition is a process which takes place directly after the ICM organoid composition from single cells. Propagating in time, cell division leads to a local clustering of cell fates and a cell sorting process yields the final separation of PrE and Epi cells. Krupinski et al. (2011) hypothesised differential adhesion between cells of different fates as a critical determinant in the robust ICM formation (49).

\section{Spatial analysis of expression type distribution}

NANOG and GATA6 are described as the first markers for Epi and PrE cell fate segregation, with a salt-and-pepper like occurrence in early and mid mouse blastocyst. During blastocyst growth, PrE cells are sorted to the rim of the ICM, while Epi cells remain in the centre of the ICM (1, 7, 10-17). This behaviour of PrE and Epi cells is reflected in ICM organoids. While $\mathrm{N}_{+} \mathrm{G}_{-}$and $\mathrm{N}_{-} \mathrm{G}_{+}$cells are evenly distributed over the $24 \mathrm{~h}$ old ICM organoid, they re-localise in $48 \mathrm{~h}$ old ICM organoids, forming a centre of $\mathrm{N}_{+} \mathrm{G}_{-}$cells and a rim of $\mathrm{N}_{-} \mathrm{G}_{+}$cells. $\mathrm{N}_{+} \mathrm{G}_{+}$cells are co-expressing NANOG and GATA6, as described for early mouse blastocysts $(\mathrm{E} 3.0)(7,11,12)$. Thus, we expected them to be distributed evenly over the whole ICM for $24 \mathrm{~h}$ old 
and $48 \mathrm{~h}$ old ICM organoids, which was confirmed by the conducted spatial analysis. For N_G. cells, expressing low levels of NANOG and GATA6, we also expected a spatially homogeneous distribution. However, the spatial analysis revealed that N_G_ cells tended to be positioned at the rim of ICM organoids at the $24 \mathrm{~h}$ time point. This finding was surprising, but it explains the very high proportion of N_G_ cells in the neighbourhood of N_G_ cells. Our model assumptions treat all cell fates equally and do not involve a specific spatial positioning of one of the cell fates. Furthermore, we assume that the original cell fate distribution follows a salt-and-pepper pattern (following from the experimental procedure to compose the initial organoids). Apparently, a subgroup of the N.G. cells tends to assemble at the rim of the organoid. This behaviour has not been described in the literature before and is not yet understood. However, this positioning explains the disagreement between the expected neighbourhood statistics and the data for this cell type. The role and dynamics of N_G_ cells is not yet well understood, and should be investigated in further studies.

\section{Conclusions}

With this study we aimed to clarify the contributions of cell proliferation to the observed neighbourhood statistics in ICM organoids, neglecting intra- and intercellular chemical signalling as well as a cell sorting process. Assuming an initially random cell fate assignment, subsequently followed by cell divisions and cell displacement in response to mechanical cell-cell interactions, the in vitro observed neighbourhood statistics can be reproduced reasonably well.

The agreement between simulated and experimental data is best for the simplest assumptions for cell fate changes during cell division (i.e. cells strictly pass on their cell fate to daughter cells). Although the simulated neighbourhood structures fit parts of the experimental data reasonably well, the model is lacking a cell sorting process, which we hypothesise to result in the measured neighbourhood structures. Cells placed at the rim of cell colonies are described to grow faster, and thus show a higher cell division rate $(37,47)$. After the sorting of GATA6 positive cells to the rim of the ICM organoid, these cells might divide with a higher rate, which would explain the strong increase of their proportions from $24 \mathrm{~h}$ to $48 \mathrm{~h}$ old ICM organoids.

The results of our study indicate a temporal separation of the four phases shown in Fig. 3. Further indication for this assumption can be found in the work of Filimonow et al. 2019 (50). Here it has been shown that no difference in the E-cadherin levels between Epi and PrE can be found until E3.75. This implies that cell sorting does not take place until this time point, if cell sorting partially occurs due to differential adhesion $(49,51,52)$.

In particular, our results indicate that the cell fate decision takes place prior or only shortly after the organoids are assembled from 200 cells, and that the neighbourhood structure between the time points $t_{0}$ and $t_{1}$ are determined only by cell divisions and cell fate heredity. Subsequently, cell sorting starts after $t_{1}$, which is supported by (50), and eventually leads to the phase separation found $48 \mathrm{~h}$ old ICM organoids.

\section{AUTHOR CONTRIBUTIONS}

Conception and design of the study were performed by T.L. and F.M.; Experimental data were provided by B.M., S.C.F. and E.H.K.S.; T.L. implemented the code.; T.L., A.D. and F.M. analysed the data; T.L., F.M. and S.C.F. interpreted the data. T.L. wrote the manuscript. All authors revised the manuscript. F.M. supervised the study.

\section{ACKNOWLEDGMENTS}

Research in the F.M. lab is supported by the Giersch foundation. T.L., F.M. and E.H.K.S. are supported by a grant from the Hessen State Ministry for Higher Education, Research and the Arts in the framework of the Loewe Program (DynaMem). Research in the Stelzer lab is supported by the Deutsche Forschungsgemeinschaft (CEF-MC II, EXC-115). B.M. has additionally been supported by fellowships from the Joachim Herz Stiftung and Freunde und Förderer der Goethe-Universität. S.C.F. and E.H.K.S. have been supported by an International Exchanges Grant from The Royal Society (IE141022).

\section{REFERENCES}

1. Rivera-Pérez, J. A., and A.-K. Hadjantonakis, 2015. The dynamics of morphogenesis in the early mouse embryo. Cold Spring Harbor perspectives in biology 7:a015867.

2. Downs, K. M., and T. Davies, 1993. Staging of gastrulating mouse embryos by morphological landmarks in the dissecting microscope. Development 118:1255-1266.

3. Wilcox, A. J., C. R. Weinberg, J. F. O'connor, D. D. Baird, J. P. Schlatterer, R. E. Canfield, E. G. Armstrong, and B. C. Nisula, 1988. Incidence of early loss of pregnancy. New England Journal of Medicine 319:189-194. 
4. Tarkowski, A. K., and J. Wróblewska, 1967. Development of blastomeres of mouse eggs isolated at the 4-and 8-cell stage. Development 18:155-180.

5. Johnson, M. H., and J. M. McConnell, 2004. Lineage allocation and cell polarity during mouse embryogenesis. In Seminars in cell \& developmental biology. Elsevier, volume 15, 583-597.

6. Dziadek, M., 1979. Cell differentiation in isolated inner cell masses of mouse blastocysts in vitro: onset of specific gene expression. Development 53:367-379.

7. Chazaud, C., Y. Yamanaka, T. Pawson, and J. Rossant, 2006. Early lineage segregation between epiblast and primitive endoderm in mouse blastocysts through the Grb2-MAPK pathway. Developmental cell 10:615-624.

8. Mihajlović, A. I., V. Thamodaran, and A. W. Bruce, 2015. The first two cell-fate decisions of preimplantation mouse embryo development are not functionally independent. Scientific reports 5:15034.

9. Schrode, N., P. Xenopoulos, A. Piliszek, S. Frankenberg, B. Plusa, and A.-K. Hadjantonakis, 2013. Anatomy of a blastocyst: cell behaviors driving cell fate choice and morphogenesis in the early mouse embryo. Genesis 51:219-233.

10. Ohnishi, Y., W. Huber, A. Tsumura, M. Kang, P. Xenopoulos, K. Kurimoto, A. K. Oleś, M. J. Araúzo-Bravo, M. Saitou, A.-K. Hadjantonakis, et al., 2014. Cell-to-cell expression variability followed by signal reinforcement progressively segregates early mouse lineages. Nature cell biology 16:27.

11. Plusa, B., A. Piliszek, S. Frankenberg, J. Artus, and A.-K. Hadjantonakis, 2008. Distinct sequential cell behaviours direct primitive endoderm formation in the mouse blastocyst. Development 135:3081-3091.

12. Zernicka-Goetz, M., S. A. Morris, and A. W. Bruce, 2009. Making a firm decision: multifaceted regulation of cell fate in the early mouse embryo. Nature Reviews Genetics 10:467.

13. Singh, A. M., T. Hamazaki, K. E. Hankowski, and N. Terada, 2007. A heterogeneous expression pattern for Nanog in embryonic stem cells. Stem cells 25:2534-2542.

14. Guo, G., M. Huss, G. Q. Tong, C. Wang, L. L. Sun, N. D. Clarke, and P. Robson, 2010. Resolution of cell fate decisions revealed by single-cell gene expression analysis from zygote to blastocyst. Developmental cell 18:675-685.

15. Frankenberg, S., F. Gerbe, S. Bessonnard, C. Belville, P. Pouchin, O. Bardot, and C. Chazaud, 2011. Primitive endoderm differentiates via a three-step mechanism involving Nanog and RTK signaling. Developmental cell 21:1005-1013.

16. Kang, M., P. Xenopoulos, S. Muñoz-Descalzo, X. Lou, and A.-K. Hadjantonakis, 2013. Live imaging, identifying, and tracking single cells in complex populations in vivo and ex vivo. In Imaging and Tracking Stem Cells, Springer, 109-123.

17. Schrode, N., N. Saiz, S. Di Talia, and A.-K. Hadjantonakis, 2014. GATA6 levels modulate primitive endoderm cell fate choice and timing in the mouse blastocyst. Developmental cell 29:454-467.

18. Takaoka, K., and H. Hamada, 2012. Cell fate decisions and axis determination in the early mouse embryo. Development 139:3-14.

19. Yamanaka, Y., F. Lanner, and J. Rossant, 2010. FGF signal-dependent segregation of primitive endoderm and epiblast in the mouse blastocyst. Development 137:715-724.

20. Hermitte, S., and C. Chazaud, 2014. Primitive endoderm differentiation: from specification to epithelium formation. Philosophical Transactions of the Royal Society B: Biological Sciences 369:20130537.

21. Cai, K. Q., C. D. Capo-Chichi, M. E. Rula, D.-H. Yang, and X.-X. Xu, 2008. Dynamic GATA6 expression in primitive endoderm formation and maturation in early mouse embryogenesis. Developmental Dynamics 237:2820-2829.

22. Silva, J., J. Nichols, T. W. Theunissen, G. Guo, A. L. van Oosten, O. Barrandon, J. Wray, S. Yamanaka, I. Chambers, and A. Smith, 2009. Nanog is the gateway to the pluripotent ground state. Cell 138:722-737.

23. Messerschmidt, D. M., and R. Kemler, 2010. Nanog is required for primitive endoderm formation through a non-cell autonomous mechanism. Developmental biology 344:129-137. 
24. Artus, J., and C. Chazaud, 2014. A close look at the mammalian blastocyst: epiblast and primitive endoderm formation. Cellular and molecular life sciences 71:3327-3338.

25. Bessonnard, S., L. De Mot, D. Gonze, M. Barriol, C. Dennis, A. Goldbeter, G. Dupont, and C. Chazaud, 2014. Gata6, Nanog and Erk signaling control cell fate in the inner cell mass through a tristable regulatory network. Development 141:3637-3648.

26. Schröter, C., P. Rué, J. P. Mackenzie, and A. M. Arias, 2015. FGF/MAPK signaling sets the switching threshold of a bistable circuit controlling cell fate decisions in embryonic stem cells. Development 142:4205-4216.

27. De Mot, L., D. Gonze, S. Bessonnard, C. Chazaud, A. Goldbeter, and G. Dupont, 2016. Cell fate specification based on tristability in the inner cell mass of mouse blastocysts. Biophysical journal 110:710-722.

28. Foty, R. A., C. M. Pfleger, G. Forgacs, and M. S. Steinberg, 1996. Surface tensions of embryonic tissues predict their mutual envelopment behavior. Development 122:1611-1620.

29. Meilhac, S. M., R. J. Adams, S. A. Morris, A. Danckaert, J.-F. Le Garrec, and M. Zernicka-Goetz, 2009. Active cell movements coupled to positional induction are involved in lineage segregation in the mouse blastocyst. Developmental biology 331:210-221.

30. Moore, R., K. Q. Cai, D. O. Escudero, and X.-X. Xu, 2009. Cell adhesive affinity does not dictate primitive endoderm segregation and positioning during murine embryoid body formation. Genesis 47:579-589.

31. Moore, R., W. Tao, Y. Meng, E. R. Smith, and X.-X. Xu, 2014. Cell adhesion and sorting in embryoid bodies derived from N-or E-cadherin deficient murine embryonic stem cells. Biology open 3:121-128.

32. McDole, K., Y. Xiong, P. A. Iglesias, and Y. Zheng, 2011. Lineage mapping the pre-implantation mouse embryo by two-photon microscopy, new insights into the segregation of cell fates. Developmental biology 355:239-249.

33. Watanabe, T., J. S. Biggins, N. B. Tannan, and S. Srinivas, 2014. Limited predictive value of blastomere angle of division in trophectoderm and inner cell mass specification. Development 141:2279-2288.

34. Anani, S., S. Bhat, N. Honma-Yamanaka, D. Krawchuk, and Y. Yamanaka, 2014. Initiation of Hippo signaling is linked to polarity rather than to cell position in the pre-implantation mouse embryo. Development 141:2813-2824.

35. Mathew, B., S. Muñoz-Descalzo, E. Corujo-Simon, C. Schröter, E. H. Stelzer, and S. C. Fischer, 2019. Mouse ICM organoids reveal three-dimensional cell fate clustering. Biophysical journal 116:127-141.

36. Karolak, A., D. A. Markov, L. J. McCawley, and K. A. Rejniak, 2018. Towards personalized computational oncology: from spatial models of tumour spheroids, to organoids, to tissues. Journal of The Royal Society Interface 15:20170703.

37. Drasdo, D., and S. Höhme, 2005. A single-cell-based model of tumor growth in vitro: monolayers and spheroids. Physical biology 2:133.

38. Drasdo, D., S. Hoehme, and M. Block, 2007. On the role of physics in the growth and pattern formation of multi-cellular systems: what can we learn from individual-cell based models? Journal of Statistical Physics 128:287.

39. Germann, P., M. Marin-Riera, and J. Sharpe, 2019. yal|a: GPU-Powered Spheroid Models for Mesenchyme and Epithelium. Cell Systems 8:261 - 266.

40. Rejniak, K. A., and A. R. Anderson, 2011. Hybrid models of tumor growth. Wiley Interdisciplinary Reviews: Systems Biology and Medicine 3:115-125.

41. Tosenberger, A., D. Gonze, S. Bessonnard, M. Cohen-Tannoudji, C. Chazaud, and G. Dupont, 2017. A multiscale model of early cell lineage specification including cell division. NPJ systems biology and applications 3:16.

42. Stichel, D., A. M. Middleton, B. F. Müller, S. Depner, U. Klingmüller, K. Breuhahn, and F. Matthäus, 2017. An individual-based model for collective cancer cell migration explains speed dynamics and phenotype variability in response to growth factors. NPJ systems biology and applications 3:5.

43. Saiz, N., and B. Plusa, 2013. Early cell fate decisions in the mouse embryo. Reproduction 145:R65-R80. 
44. Elowitz, M. B., A. J. Levine, E. D. Siggia, and P. S. Swain, 2002. Stochastic gene expression in a single cell. Science 297:1183-1186.

45. Dietrich, J.-E., and T. Hiiragi, 2007. Stochastic patterning in the mouse pre-implantation embryo. Development 134:4219-4231.

46. Simon, C. S., A.-K. Hadjantonakis, and C. Schröter, 2018. Making lineage decisions with biological noise: Lessons from the early mouse embryo. Wiley Interdisciplinary Reviews: Developmental Biology 7:e319.

47. Streichan, S. J., C. R. Hoerner, T. Schneidt, D. Holzer, and L. Hufnagel, 2014. Spatial constraints control cell proliferation in tissues. Proceedings of the National Academy of Sciences 111:5586-5591.

48. Krens, S. G., and C.-P. Heisenberg, 2011. Cell sorting in development. In Current topics in developmental biology, Elsevier, volume 95, 189-213.

49. Krupinski, P., V. Chickarmane, and C. Peterson, 2011. Simulating the mammalian blastocyst-molecular and mechanical interactions pattern the embryo. PLoS computational biology 7:e1001128.

50. Filimonow, K., N. Saiz, A. Suwińska, T. Wyszomirski, J. B. Grabarek, E. Ferretti, A. Piliszek, B. Plusa, and M. Maleszewski, 2019. No evidence of involvement of E-cadherin in cell fate specification or the segregation of Epi and PrE in mouse blastocysts. PloS one 14:e0212109.

51. Steinberg, M. S., 2007. Differential adhesion in morphogenesis: a modern view. Current opinion in genetics \& development 17:281-286.

52. Nissen, S. B., M. Perera, J. M. Gonzalez, S. M. Morgani, M. H. Jensen, K. Sneppen, J. M. Brickman, and A. Trusina, 2017. Four simple rules that are sufficient to generate the mammalian blastocyst. PLoS biology 15:e2000737.

An online supplement to this article can be found by visiting BJ Online at http://www . biophysj . org. 

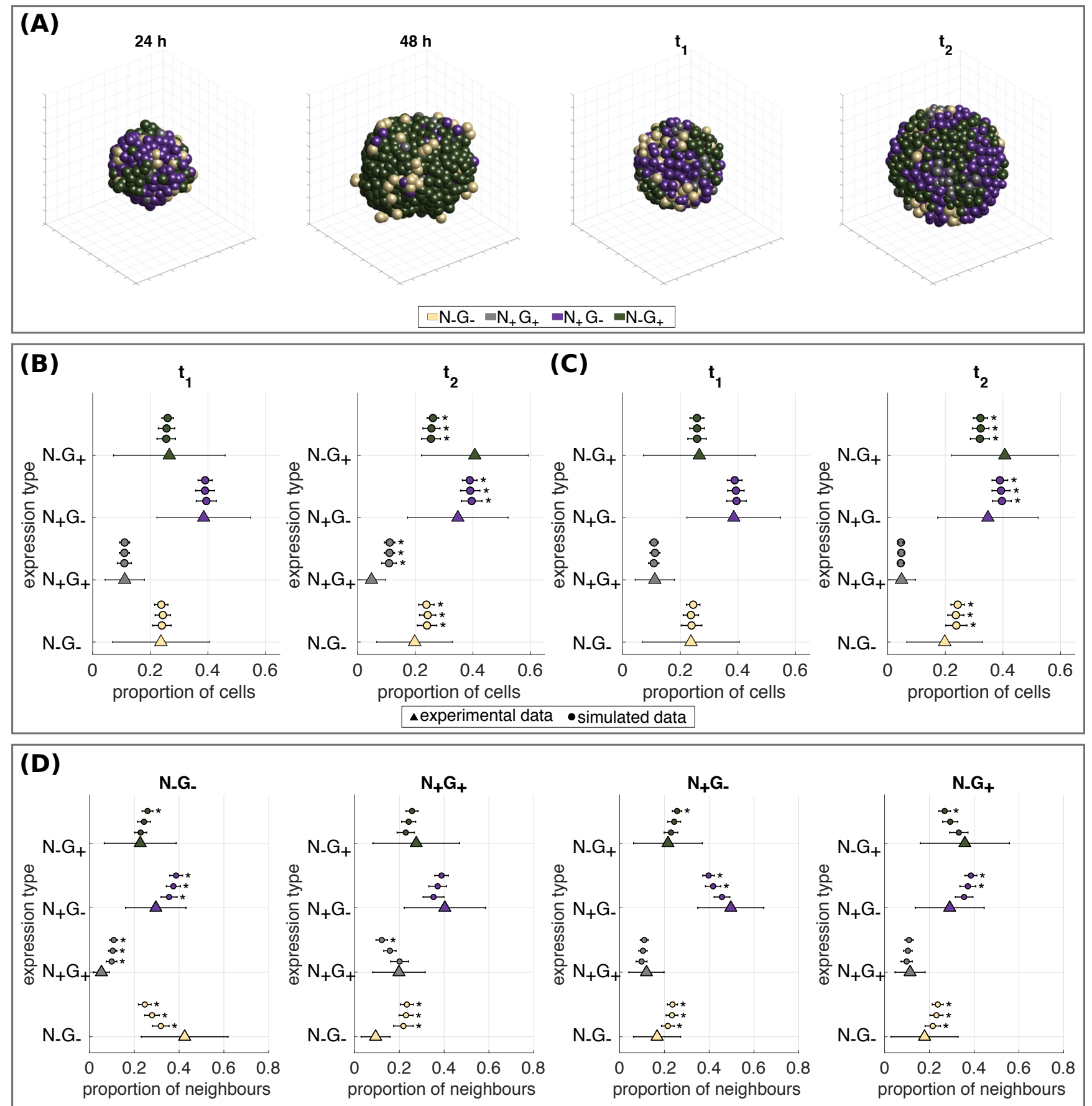

(E)
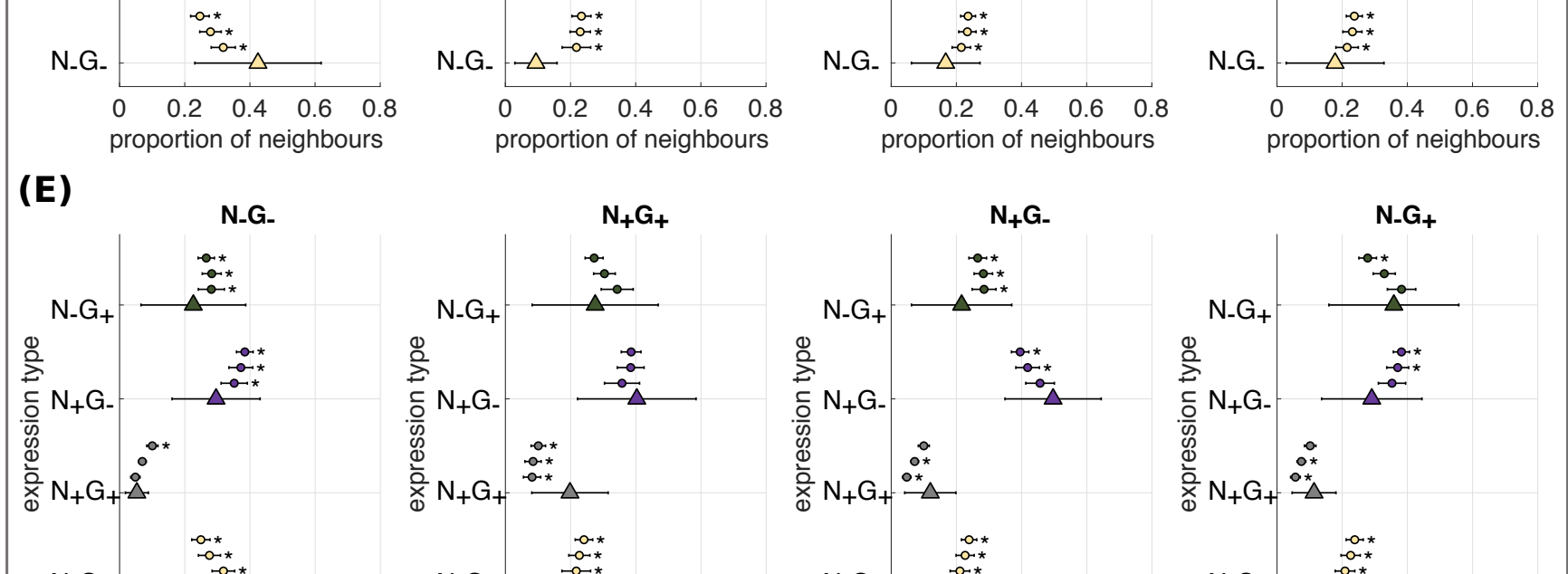

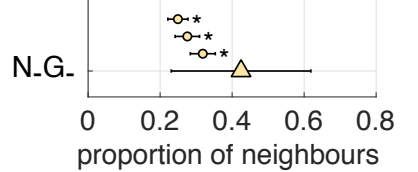

$$
\begin{aligned}
& \begin{array}{lllll}
0 & 0.2 & 0.4 & 0.6 & 0.8 \\
\text { proportion of neighbours }
\end{array}
\end{aligned}
$$

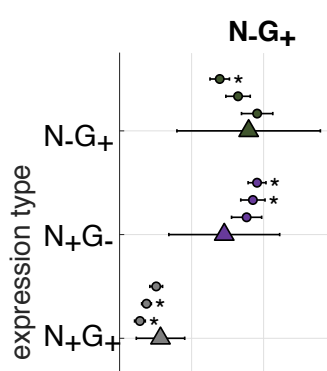


bioRxiv preprint doi: https://doi.org/10.1101/698928; this version posted February 7, 2020. The copyright holder for this preprint (which was not certified by peer review) is the author/funder. All rights reserved. No reuse allowed without permission.

Cell Fate in ICM Organoids

Figure 1: Expression type composition of ICM spheroids for H1 and H2. (A) ICM organoids (experimental data) for $24 \mathrm{~h}$ and $48 \mathrm{~h}$ and simulated ICM spheroids for $t_{1}$ and $t_{2} .(\mathrm{B}, \mathrm{C})$ Expression type composition of ICM organoids and ICM spheroids shown as percentage of the total number of cells within ICM organoids at $t_{1}$ and $t_{2}$. Statistically significant differences between the cell fate proportion of ICM organoids and ICM spheroids are indicated by stars $(p<0.05$; using a Wilcoxon-Mann-Whitney test with Bonferroni correction). Simulations were performed under the assumption H1 (B) and the assumption H2 (C). (D, E) Expression type composition of neighbouring cells shown as percentage of the total of neighbouring cells at $t_{1}$. Simulations were performed under the assumption H1 (D) and the assumption H2 (E). Experimental data from Mathew et al. (2019) are indicated by triangles. Simulation results for different $t_{0}$ are indicated by circles. The error bars indicate the standard deviation. $t_{0}$ from lowest line to top: 200,300 and 400 cells. Statistically significant differences between the neighbourhood structure of $24 \mathrm{~h}$ old ICM organoids and ICM spheroid patterns are indicated by stars $(p<0.05$; using a Wilcoxon-Mann-Whitney test with Bonferroni correction). 


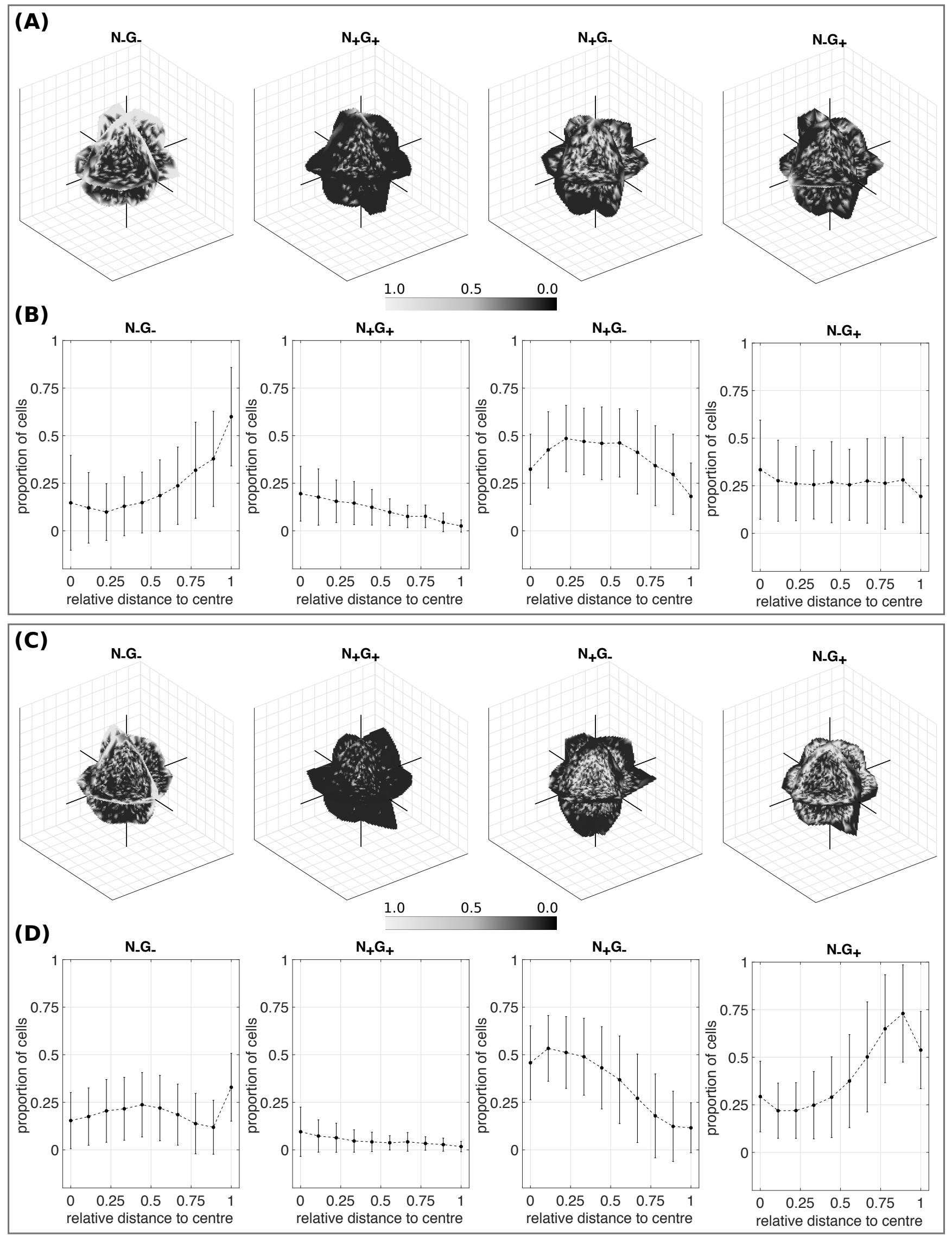

Figure 2 
bioRxiv preprint doi: https://doi.org/10.1101/698928; this version posted February 7, 2020. The copyright holder for this preprint (which was not certified by peer review) is the author/funder. All rights reserved. No reuse allowed without permission.

Cell Fate in ICM Organoids

Figure 2: Expression type cluster analysis for ICM organoids. (A) $24 \mathrm{~h}$ old ICM organoids; (C) $48 \mathrm{~h}$ old ICM organoids. Black indicates the absence of an expression type, white indicates the presence of the selected expression type, respectively. Shown are slices through the ICM organoids at $x=0, y=0$ and $z=0$. (B, D) Expression type compositions in dependence of the relative distance to the ICM organoid centre for $24 \mathrm{~h}$ old ICM organoids (B) and for $48 \mathrm{~h}$ old ICM organoids (D). Cells are sorted according to their distance to the ICM organoid centre of mass and binned into 10 groups. Points indicate the mean proportion of a cell fate type for the 10 bins, the bars denote the standard deviation. 
Tim Liebisch

(A)

coexpression

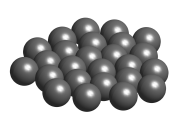

(B) initial cell fate assignment

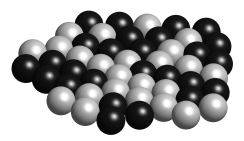

(C)

cell division leads to local clustering

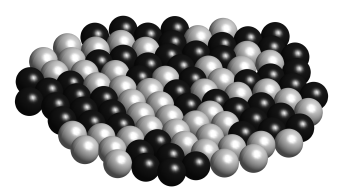

(D)

\section{cell sorting} process

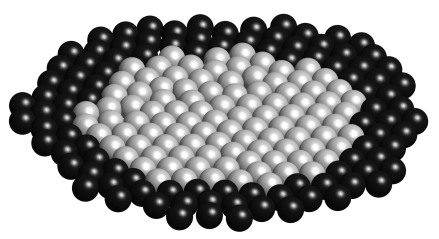

\section{time}

Figure 3: Working model. Over time, four phases lead to the separation of GATA6 positive (black) and NANOG positive (white) cells. Initially, all cells coexpress both cell fate markers (A), followed by a random cell fate assignment (B). Cell division leads to a local clustering of cells sharing the same cell fate $(\mathrm{C})$ and eventually a sorting mechanism leads to a phase separation of GATA6 positive and NANOG positive cells. 